\title{
Extension of Lighthill's slender-body theory to moderate aspect ratios
}

\author{
Zhanle Yu, Christophe Eloy \\ Aix-Marseille Univ, CNRS, Centrale Marseille, IRPHE, Marseille, France
}

\begin{abstract}
Calculating the fluid forces acting on a moving body at high Reynolds number is crucial in many fluidstructure interaction problems, such as fish swimming or flutter instabilities. To estimate these forces, Lighthill developed the slender-body theory, which assumes a potential flow and an asymptotically small aspect ratio. Yet, it is still unclear whether Lighthill's theory is still valid for aspect ratios of order one. To address this question, we solve numerically with a panel method the full three-dimensional problem of a rectangular plate deforming periodically in a potential flow. These numerical simulations are used to calculate the pressure jump distribution across the plate for different aspect ratios. We find that numerical simulations and slender-body theory give similar results far from trailing edge. Close to the trailing edge however, there is a discrepancy, which is due to the use of a Kutta condition in the simulations (i.e. no pressure jump at the trailing edge), while, in the slender-body theory, the pressure jump is non zero. We propose a simple extension of Lighthill's slender-body theory that accounts for this discrepancy. The usefulness of this extension is then discussed and illustrated with a generic fluid-structure interaction problem and with the flag instability problem.
\end{abstract}

Keywords: Slender-body theory, Vortex panel method, Flutter instability

\section{Introduction}

Slender-body theory has first been developed by Munk (1924) to study aerodynamic loads exerted on an airship hull. Munk (1924) considered an elongated rigid body moving in a fluid at rest. This theory is essentially based on potential flow theory and conservation of fluid momentum along the body axis. It implicitly assumes that the flow generated by each section does not affect the other sections of the body, which is guaranteed if the body is sufficiently slender and if the section does not vary abruptly.

In the context of fish swimming, Lighthill (1960) later extended slender-body theory to calculate the fluid forces exerted on a deforming body. In the present paper, we will refer to this extension as Lighthill's slender-body theory, or simply slender-body theory when there is no ambiguity. Its hypotheses are the same as in Munk (1924): the flow is assumed to be potential and incompressible, the body aspect ratio, defined as the ratio between crosswise and streamwise dimensions, is assumed to be asymptotically small. With these hypotheses, again, pressure forces exerted on the body can be calculated using an argument of momentum conservation. Remarkably, Lighthill (1960) showed that when a fish deforms periodically, the average total force only depends on the kinematics of the tail.

Slender-body theory is limited to displacements that are small compared to the streamwise body length. To circumvent this limitation, Lighthill (1971) extended slender-body theory, a theory known as Lighthill's elongated-body theory. Recently, this elongated-body theory was itself extended by Candelier et al. (2011) and Candelier et al. (2013) to respectively account for arbitrary three-dimensional motions (including all components of rotation and translation) and a non-uniform background flow.

An important field of application of Lighthill's slender-body and elongated-body theories has been the hydrodynamics of fish swimming, as first advocated by Lighthill (1960, 1971). In this context, these theories have been used to better understand the kinematics and energetics of swimming, and in particular to assess whether fish employ smart drag reduction mechanisms or if, on the contrary, drag is enhanced by fish motions 
(Alexander, 1977; Webb, 1975; Videler, 1981; Ehrenstein and Eloy, 2013). To give an unbiased review of the field, we should also mention that some authors criticized Lighthill's theories, arguing that these potential approaches tend to overestimate forces (Hess and Videler, 1984; Anderson et al., 2001; Shirgaonkar et al., 2009). Lighthill's slender-body has also been applied to engineering problems, such as the flutter instability (Lemaitre et al., 2005), or energy harvesting (Singh et al., 2012; Michelin and Doaré, 2013). Note that Lighthill's slender-body theory should not be confused with the low-Reynolds-number slender-body theory (e.g., Lauga and Powers, 2009), even if - to add to the confusion - Lighthill also contributed to this field (Lighthill, 1976).

A significant limitation of Lighthill's slender-body theory is that it is only applicable to bodies of asymptotically small aspect ratios. In the context of the flutter instability, one of us addressed this limitation by developing a spectral approach (Eloy et al., 2007, 2008). In essence, the potential flow problem is first solved in the Fourier space for any aspect ratio; then, an inverse Fourier transform is applied to come back to real space. Unfortunately, this approach is limited to body with a rectangular planform and its implementation is not as simple as Lighthill's slender-body theory. The objective of the present study is to examine a alternative analytical method to treat bodies with moderate aspect ratios.

Another important drawback of Lighthill's slender-body theory is that it is not compatible with a Kutta condition at the trailing edge. Indeed, the Kutta condition (Crighton, 1985), even for unsteady flows, requires that the pressure jump across the lifting surface is zero at the trailing edge. In Lighthill's slenderbody theory, not only the force at the trailing edge is finite, but its value is the only relevant quantity if one is interested in the average total force for a periodic motion.

This paper is organized as follows. In $\S 2$, we will recall how the lifting-surface problem is stated and we will describe an asymptotic solution of this problem valid in the limit of small aspect ratios. In $\S 3$, we will present a panel method in the frequency domain, aimed at solving the same problem numerically. An alternative analytical approach, also valid in the limit of small aspect ratios and called the approximate solution, will then be presented in $\S 4$. The mathematical derivation of this approximate solution may be less rigorous than the derivation of the asymptotic solution, but it is much simpler to implement and it provides an straightforward and useful correction to slender-body theory. The merits of these two different methods will be compared to slender-body theory and to the numerical panel method in $\S 5$ through a generic fluid-structure interaction problem and through the analysis of the flutter instability of a cantilever flexible plate. Finally, these results will be summarized and discussed in $\S 6$.

\section{Asymptotic solution of the lifting-surface integral}

Consider a uniform, incompressible, and irrotional flow of velocity $\mathbf{U}$ over a flexible plate deforming periodically with angular frequency $\Omega$ (Fig. 1). In a Cartesian coordinate system $(O X Y Z)$, the incoming fluid of density $\rho$ is flowing along $X$, and the flexible surface is deforming around the reference plane $Z=0$. The periodic deformation of the plate is described by the complex displacement $Z\left(M_{0}, T\right)=h\left(M_{0}\right) e^{i \Omega T}$, where $M_{0}$ is a point in the plane $Z=0$. The displacement is assumed sufficiently small such that (1) vortex sheets attached to the flexible surface and shed in the wake can be represented by their projections onto the reference plane, respectively $S$ and $\Sigma$ (Fig. 1); (2) the flow induced by the motion of the flexible plate can be considered as a periodic perturbation of the incident flow. For the sake of simplicity, the complex factor $e^{i \Omega T}$ will be omitted for all unsteady quantities. The point $M_{0}$ will represent an arbitrary point in the plane $S$, the point $M$ will be in $S$ or $\Sigma$, and $Q$ will be a point in the volume, outside $S$ and $\Sigma$.

Because we assume a potential flow, a perturbation potential $\Phi$ can be defined such that the total velocity field is given by $\mathbf{U}+\nabla \Phi$. Because the flow is incompressible, $\Phi$ obeys a Laplace equation with a kinematic Neumann boundary condition given by the impermeability on the plate

$$
\Delta \Phi(Q)=0, \quad \text { with } \quad \partial_{Z} \Phi\left(M_{0}\right)=W\left(M_{0}\right)=D\left(h\left(M_{0}\right)\right), \quad \text { and } D=i \Omega+U \partial_{X},
$$

where $W$ is the imposed $Z$-component of the flow velocity, called the downwash, and $D$ is the convective derivative. Note that the $Z$-derivative of $\Phi$ has no definition on $S$, hence, we define

$$
\partial_{Z} \Phi\left(M_{0}\right)=\lim _{Z_{Q} \rightarrow 0^{ \pm}} \partial_{Z} \Phi(Q)
$$




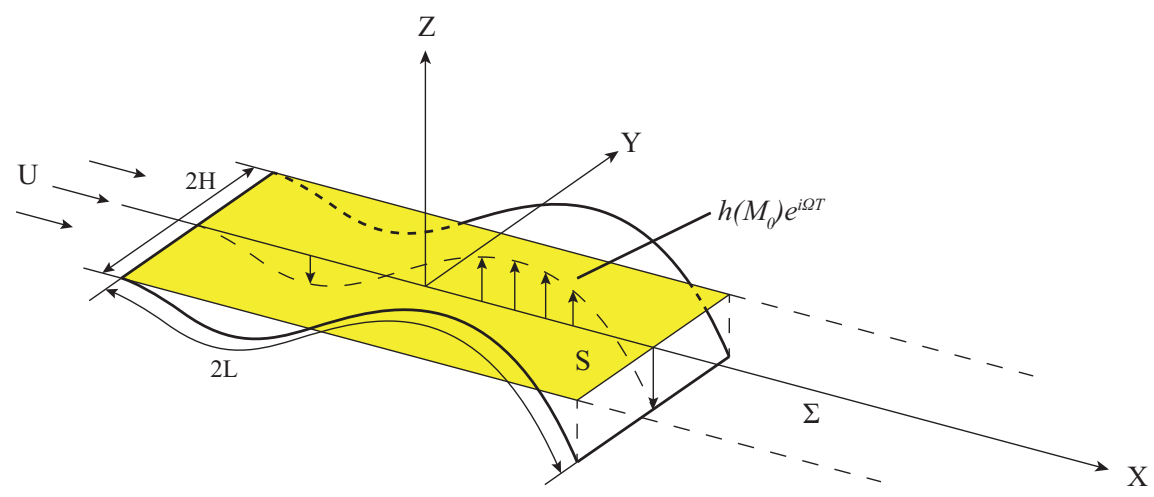

Figure 1: Schematic representation of the lifting-surface problem. A flexible plate, with chord $2 L$ and span $2 H$, is deforming periodically in an incoming flow $\mathbf{U}$.

where $M_{0}$ is the projection of $Q$ onto the reference plane. Physically, this definition simply states that the $Z$-component of flow velocity is a continuous function, including in $Z=0$.

Equation (1) can be solved by means of the Green's representation theorem (Morse and Feshbach, 1953). For any point $Q$, the potential $\Phi$ can be written as

$$
\Phi(Q)=-\int_{S+\Sigma}[\Phi](M) \frac{\partial}{\partial Z_{M}} G(M, Q) \mathrm{d} S_{M},
$$

where $[\Phi]$ is the jump of $\Phi$ across $S$, and $G(M, Q)$ is the Green's function of the three-dimensional Laplace equation:

$$
\begin{aligned}
& {[\Phi](M)=\Phi\left(X_{M}, Y_{M}, 0^{+}\right)-\Phi\left(X_{M}, Y_{M}, 0^{-}\right),} \\
& G(M, Q)=-\frac{1}{4 \pi|M Q|} .
\end{aligned}
$$

The Neumann boundary condition is imposed by applying $Z$-derivative operator onto Eq. (3) and taking the limit $Z_{Q} \rightarrow 0^{ \pm}$. This yields

$$
W\left(M_{0}\right)=\lim _{Z_{Q} \rightarrow 0^{ \pm}} \int_{S+\Sigma}[\Phi](M) \frac{\partial^{2}}{\partial Z_{Q} \partial Z_{M}} G(M, Q) \mathrm{d} S_{M},
$$

where $M_{0}$ is the projection of $Q$ onto the reference plane. The existence of this limit is guaranteed physically, and it can be evaluated by exchanging the limit and integral operators

$$
W\left(M_{0}\right)=\oint_{S+\Sigma}[\Phi](M) K\left(M, M_{0}\right) \mathrm{d} S_{M},
$$

with

$$
K\left(M, M_{0}\right)=\lim _{Z_{Q} \rightarrow 0^{ \pm}} \frac{\partial^{2}}{\partial Z_{Q} \partial Z_{M}} G(M, Q)=\frac{1}{4 \pi\left|M M_{0}\right|^{3}} .
$$

The above exchange of operators is only permitted when the integral is taken in the finite-part sense of Hadamard (1923) (represented by the cross on the integral sign). In this case, the singular integral in Eq. (7), usually called the lifting-surface integral exists and can, in principle, be calculated, as it has been shown by Mangler (1951) and Brandao (1987).

Assuming the potential jump $[\Phi]$, solution of Eq. (7), is found, the pressure jump across the plate, $[P]$, can be calculated with the linearized unsteady Bernoulli equation

$$
[P]\left(M_{0}\right)=-\rho D[\Phi]\left(M_{0}\right) .
$$


The downwash $W$ is related to the unknown potential jump $[\Phi]$ through a lifting-surface integral $(7)$. Mathematically, this is a Fredholm integral equation of the first kind with a hypersingular kernel $K\left(\left|M M_{0}\right|\right)$. The inversion of Eq. (7) is the essential part of a lifting-surface problem. For a lifting surface of arbitrary geometry, the analytic inversion is generally impossible. In this case, numerical techniques have to be used. Popular approaches include panel methods, such as the vortex-lattice method (Katz and Plotkin, 2001), or the doublet-lattice method (Albano and Rodden, 1969). However, if the aspect ratio of the lifting surface is asymptotically small or large, specific analytic methods such as the Matched Asymptotic Expansion can be used (Guermond, 1987; Kida and Miyai, 1978; Eloy et al., 2010).

In the limit of asymptotically small aspect ratios, the leading order and the first order correction of the force (per unit chord length) exerted by the plate onto the fluid have been explicitly given in Eloy et al. (2010)

$$
\begin{aligned}
& F_{0}(X)=D\left(\rho \pi H^{2} W(X)\right), \\
& F_{1}(X)=D\left\{\frac{\rho \pi H^{4}}{4}\left[\left(\ln \frac{4}{A}-\frac{3}{4}\right) W^{\prime \prime}(X)+\star_{-L}^{\infty} \frac{W\left(X_{M}\right)}{\left|X-X_{M}\right|^{3}} \mathrm{~d} X_{M}\right]\right\},
\end{aligned}
$$

where $H$ is the semi-span, $L$ the semi-chord, and $A=H / L$ the aspect ratio. In the following, we will refer to this approach as the asymptotic solution. The zero ${ }^{\text {th }}$ order expression in Eq. (10) is strictly equivalent to Lighthill's slender-body theory, although the derivation proposed by Lighthill (1960) is far more elegant. The factor $\left(\rho \pi H^{2}\right)$ represents the added mass of fluid per unit chord, and Eq. (10) simply expresses that the force is the rate-of-change of the fluid momentum. One of the objectives of the present paper is to assess whether the first order correction given by Eq. (11) improves the accuracy of the fluid force, especially for lifting surfaces of moderate aspect ratios. To do so, we need a reference value of the lifting force that will be computed with a panel method, as explained in the next section.

\section{Panel method in the frequency domain}

The panel method is a numerical method that can be used to invert the lifting-surface integral equation and calculate the aerodynamic loads. Since we assume here that the displacement of the moving surface is periodic and small, some simplifications can be made to reduce the computational costs. More details about the classic panel method can be found in Katz and Plotkin (2001).

First, the boundary layer and wake vortex sheet are modeled by vortex rings placed on $S$ and $\Sigma$, respectively. The impermeability condition is enforced on $S$ instead of the actual moving surface. The discretizing grid is shown in Fig. 2. The indices $i, j$, or sequential counter $k=(i-1) \times N_{s}+j$, are used to track the location of a vortex ring (or a collocation point).

Second, the unsteady quantities are expressed with the complex factor $e^{i \Omega T}$. Thus, the normal vector $\mathbf{n}_{i, j, T}$ defined at the collocation point can be written as

$$
\mathbf{n}_{i, j, T} \simeq\left(-\frac{\partial h}{\partial X} e^{i \Omega T},-\frac{\partial h}{\partial Y} e^{i \Omega T}, 1\right)
$$

The circulation of a vortex ring is equal to

$$
\Gamma_{i, j, T}=\Gamma_{i, j} e^{i \Omega T}, \quad 1 \leq i \leq N_{c}, \quad 1 \leq j \leq N_{s} .
$$

In order to fulfill the Kutta condition and enforce zero pressure jump across the wake vortex sheet $\Sigma$, the circulation of a wake vortex ring is given by

$$
\Gamma_{i, j, T}=\Gamma_{N_{c}, j} e^{i \Omega\left(T-\left(i-N_{c}\right) \Delta c / U\right)}, \quad N_{c}+1 \leq i \leq N_{w}, \quad 1 \leq j \leq N_{s},
$$

where $\Delta c$ is the chordwise length of vortex rings (Fig. 2). The above equation implies that wake vortices are first generated at the trailing edge and then advected downstream with velocity $\mathbf{U}$ without changing circulation. 


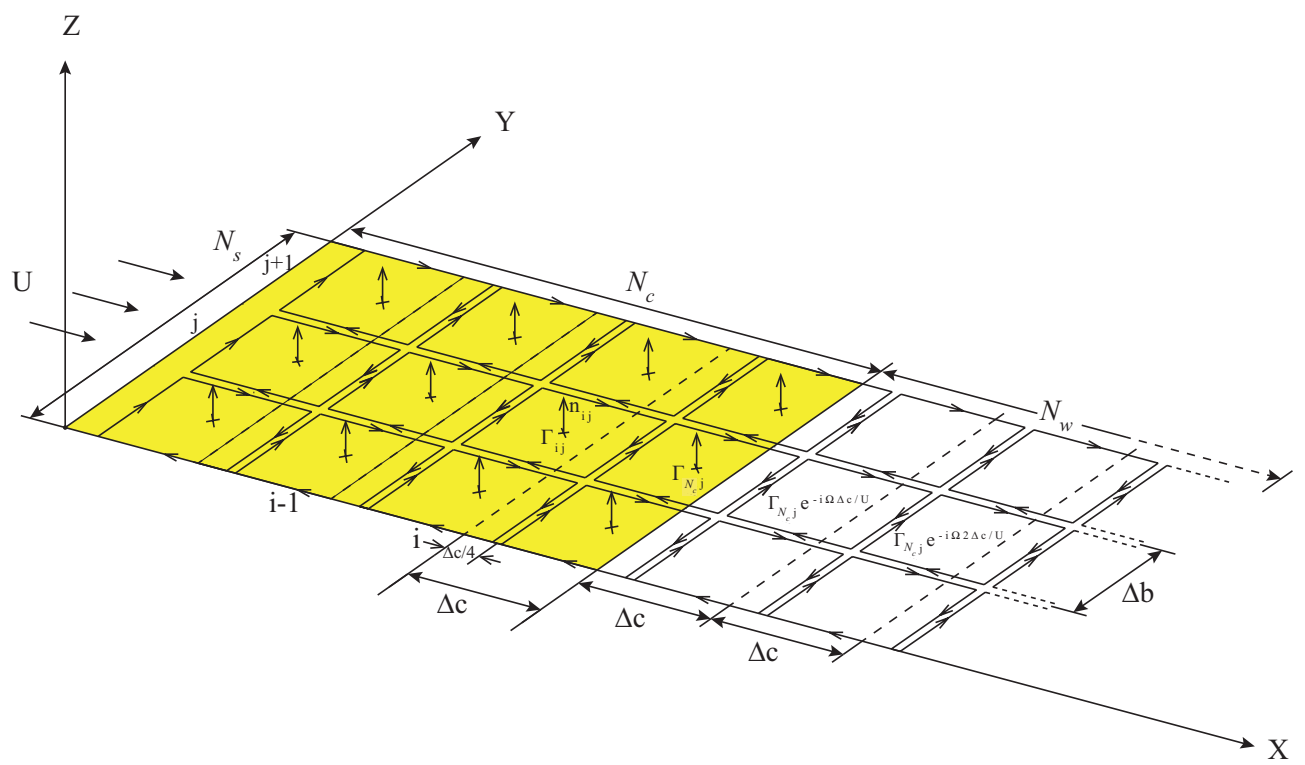

Figure 2: Representation of the numerical panel method used to solve in the frequency domain the lifting-surface equation.

As a consequence of previous simplifications, the complex factor $e^{i \Omega T}$ in the linear system can be omitted, and the impermeability condition for all collocation points on $S$ becomes independent of time. The discrete form of the system is

$$
\mathbf{M}_{a} \boldsymbol{\Gamma}=\mathbf{R},
$$

where $\mathbf{M}_{a}$ is the influence matrix, the vector $\boldsymbol{\Gamma}$ contains the unknown circulations of all vortex rings on $S$, and the elements of $\mathbf{R}$ are the values of the downwash at the collocation points (see Eq. 1). Equation (15) can be viewed as the discrete form of the lifting-surface integral (7).

The coefficients of the influence matrix $\mathbf{M}_{a}$ are given by

$$
a_{k l}= \begin{cases}a_{k l}^{*} & (\text { if } l \text { is not on the trailing edge }), \\ a_{k l}^{*}+b_{k l} & (\text { if } l \text { is on the trailing edge) }\end{cases}
$$

where the details of the coefficients $a_{k l}^{*}$ and $b_{k l}$ can be found in Katz and Plotkin (2001). The coefficients $a_{k l}^{*}$ represent the $Z$-component of the flow velocity at the collocation point $k$ induced by the vortex ring $l$ with unit circulation, and the coefficients $b_{k l}$ are the $Z$-component of the velocity induced by all wake vortex rings, which result from the advection of the trailing edge vortex ring $l$ (see Eq. 14).

The circulations of vortex rings on $S$ can be computed by inverting the linear system (15). We then calculate the pressure jump by applying a discretized version of the linearized Bernoulli equation (9). The advantage of the present approach is that the whole computation needs to be executed only once for a periodic lifting-surface problem (there is no time-marching). The computational costs are thus reduced compared to a classic unsteady panel method (Katz and Plotkin, 2001). This approach will be used to compute the aerodynamic forces exerted on a moving flexible plate. These computations will serve as reference values to assess the validity of our analytic asymptotic approaches.

\section{Approximate solution of the lifting-surface integral}

To begin with, we re-formulate the lifting-surface integral in terms of the acceleration potential $\Psi=$ $-P / \rho=D(\Phi)$ (we simply use the linearized unsteady Bernoulli equation (9)). Applying the linear differential operator $D$ to Eq. (1) yields a Laplace problem for $\Psi$ with Neumann boundary conditions

$$
\Delta \Psi(Q)=0, \quad \text { with } \quad \partial_{Z} \Psi\left(M_{0}\right)=D\left(W\left(M_{0}\right)\right)
$$


As described in section 2, the solution of Eq. (17) can be found by using the Green's representation theorem (Morse and Feshbach, 1953), and the Neumann boundary condition can be enforced by applying the $Z$-derivative operator to $\Psi$ and taking the limit $Z_{Q} \rightarrow 0$. Using this method, the lifting-surface integral is expressed in terms of the acceleration potential jump

$$
D\left(W\left(M_{0}\right)\right)=\mho_{S}[\Psi](M) K\left(\left|M M_{0}\right|\right) \mathrm{d} S_{M}
$$

where $[\Psi]$ is the acceleration potential jump across $S$, and $K$ is the kernel function given by Eq. (8). Note that, contrary to the lifting-surface integral for the pressure jump given by (7), here the integral can be taken over $S$ only, and not $S+\Sigma$, because the acceleration potential jump is zero in the wake. However, just like Eq. (7), there is no general method to invert this Fredholm integral problem. In this section, we will seek an approximate solution instead.

In order to facilitate the solving procedure, the following dimensionless variables are introduced

$$
\begin{aligned}
& x=\frac{X_{M_{0}}}{L}, \quad \xi=\frac{X_{M}}{L}, \quad y=\frac{Y_{M_{0}}}{H}, \quad \eta=\frac{Y_{M}}{H}, \\
& {[\psi]=\frac{[\Psi]}{U^{2}}, \quad w^{*}=\frac{D(W) L}{U^{2}}, \quad t=\frac{T U}{L}, \quad \omega=\frac{\Omega L}{U} .}
\end{aligned}
$$

Separating the chordwise and spanwise integration, Eq. (18) can be written

$$
w^{*}(x, y)=\frac{A}{4 \pi} \int_{-1}^{1} I(x, \epsilon) \mathrm{d} \eta,
$$

with

$$
I(x, \epsilon)=\int_{-1}^{1} \frac{[\psi](\xi, \eta)}{\left[(\xi-x)^{2}+\epsilon^{2}\right]^{3 / 2}} \mathrm{~d} \xi,
$$

where $\epsilon=A(\eta-y)$ is asymptotically small. Assume $[\psi](\xi, \eta)$ is analytic with respect to $\xi$, it can be approximated by a Taylor series

$$
[\psi](\xi, \eta)=[\psi](x, \eta)+\partial_{x}[\psi](x, \eta)(\xi-x)+\cdots
$$

Because its kernel is large when $\xi \approx x$, the integral $I(x, \epsilon)$ can be approximated by conserving only the zero $^{\text {th }}$ order term in Eq. (22), and inserting it into Eq. (21). This leads to

$$
I(x, \epsilon)=\frac{1}{\epsilon^{2}}\left(\frac{1+x}{\left[(1+x)^{2}+\epsilon^{2}\right]^{1 / 2}}+\frac{1-x}{\left[(1-x)^{2}+\epsilon^{2}\right]^{1 / 2}}\right)[\psi](x, \eta) .
$$

Noting that the pressure jump must be zero at the side edges (i.e. $[\psi](x,-1)=[\psi](x, 1)=0)$, an integration by parts of Eq. (20) yields

$$
w^{*}(x, y)=\frac{1}{4 \pi A} \int_{-1}^{1} \frac{b}{\eta-y} \partial_{\eta}[\psi](x, \eta) \mathrm{d} \eta,
$$

with

$$
b=\frac{\left[(1+x)^{2}+A^{2}(\eta-y)^{2}\right]^{1 / 2}}{1+x}+\frac{\left[(1-x)^{2}+A^{2}(\eta-y)^{2}\right]^{1 / 2}}{1-x},
$$

where the Cauchy principal value of the integral should be taken (indicated by the horizontal bar across the integral sign). The integral problem given by Eq. (24) could be inverted in the limit of small $A$. However, this would give a solution identical to the asymptotic solution presented in $\S 2$. Here, we use a different approach. We approximate this integral by first rewriting $\partial_{\eta}[\psi](x, \eta)$ as

$$
\partial_{\eta}[\psi](x, \eta)=b^{*} \partial_{\eta}\left[\psi^{*}\right](x, \eta), \quad \text { with } \quad b^{*}=2 /\left\{1+\frac{\left[(1-x)^{2}+A^{2} / 4\right]^{1 / 2}}{1-x}\right\} .
$$




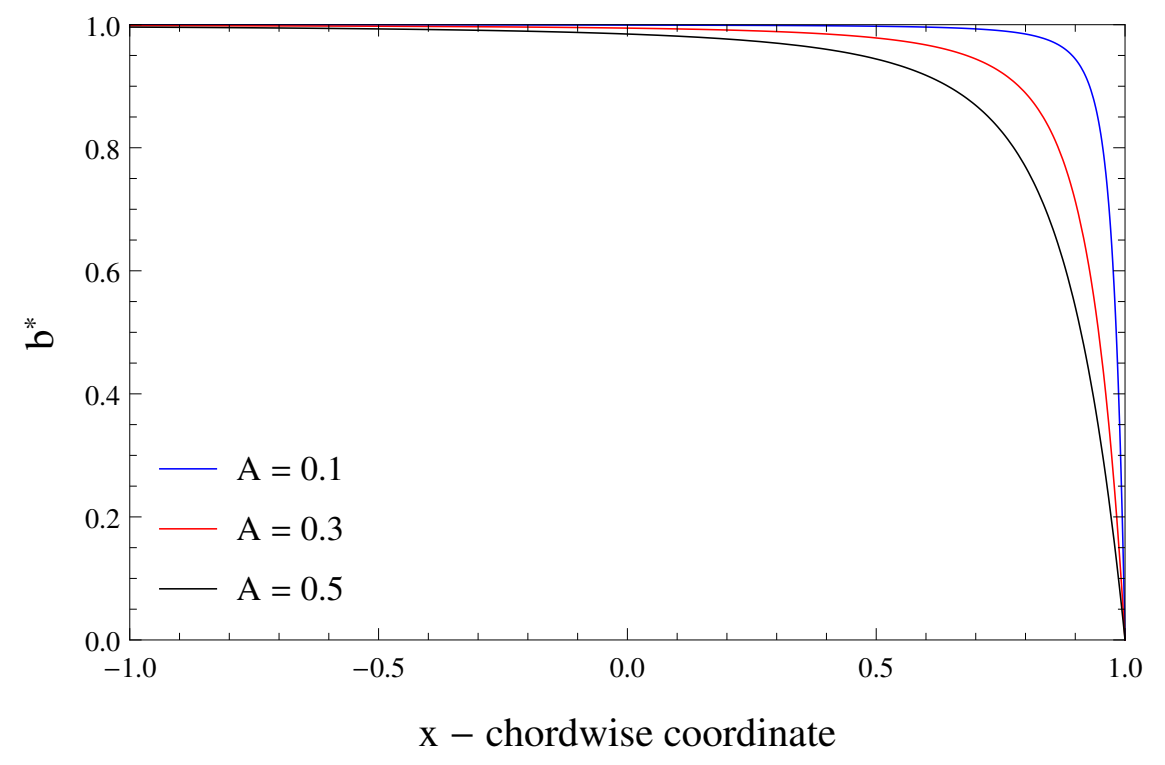

Figure 3: The factor $b^{*}$ as a function of chordwise coordinate for various aspect ratios.

The function $b^{*}(x)$ is chosen such that $b \times b^{*}$ is approximately equal to 2 , which is indeed true far from the leading edge and when $(\eta-y)^{2}$ is approximated by the value $1 / 4$ (Fig. 3). Using this approximation (i.e. $b \times b^{*} \approx 2$ ) and inserting it into Eq. (24), the integral problem is transformed into

$$
w^{*}(x, y) \simeq \frac{1}{2 \pi A} \int_{-1}^{1} \frac{1}{\eta-y} \partial_{\eta}\left[\psi^{*}\right](x, \eta) \mathrm{d} \eta .
$$

The above manipulations and approximations have led to an inverse problem where the kernel no longer depends on $x$ and the unknown no longer depends on $y$. Equation (27) is a Carleman-type singular integral equation (Carleman, 1922) that can be inverted and whose solution $\partial_{\eta}\left[\psi^{*}\right](x, \eta)$ is given by (Muskhelishvili, 1958; Tuck, 1980)

$$
\partial_{y}\left[\psi^{*}\right](x, y)=-\frac{2 A}{\pi \sqrt{1-y^{2}}} \int_{-1}^{1} \frac{\sqrt{1-\eta^{2}} w^{*}(x, \eta)}{\eta-y} \mathrm{~d} \eta+\frac{C_{0}}{\sqrt{1-y^{2}}},
$$

where the constant $C_{0}$ can be found by enforcing that the pressure jump is zero at the side edges. Integrating Eq. (28), using Eq. (26), and integrating with respect to $y$, the pressure jump $[\psi]$ can be calculated. This approximate solution of the lifting-surface integral equation will be referred to as the approximate solution.

To give a clearer view of the solving procedure, we will consider a particular case: a flexible surface whose displacement only depends on $x$ (i.e. $w^{*}$ is a function of $x$ only). Using Eq. (28) and the boundary condition on the side edges, we obtain

$$
\partial_{y}[\psi](x, y)=2 A b^{*} w^{*}(x) \frac{y}{\sqrt{1-y^{2}}}
$$

And a simple integration gives the leading-order acceleration potential jump, which can be written in dimensional terms as

$$
[P](X, Y)=2 \rho b^{*} D(W(X)) \sqrt{H^{2}-Y^{2}} .
$$

Now integrating Eq. (30) between $-H$ and $H$ gives the force per unit chord length exerted on the fluid

$$
F_{0}^{a}(X)=b^{*} \rho \pi H^{2} D(W(X))
$$

This equation is very similar to the results of Lighthill's slender-body theory, recalled in Eq. (10), except for the factor $b^{*}$, which is plotted in Fig. 3 as a function of $x$. It can readily be seen that $b^{*}$ is approximately 1 
(a)

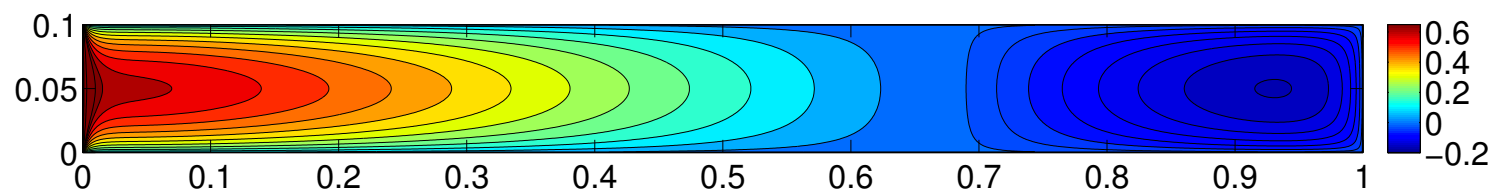

(b)

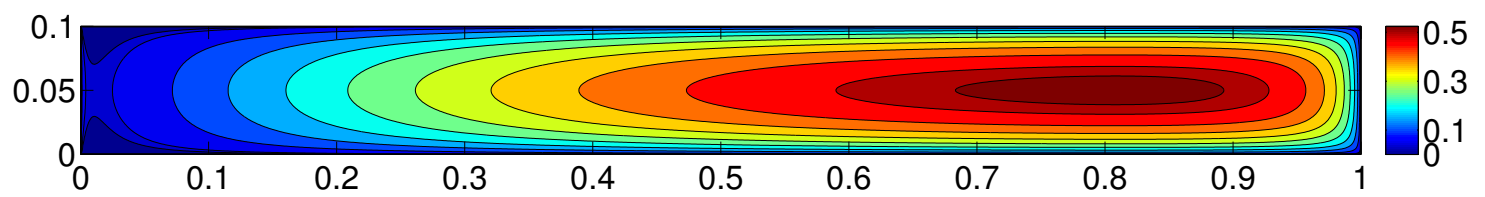

(c)

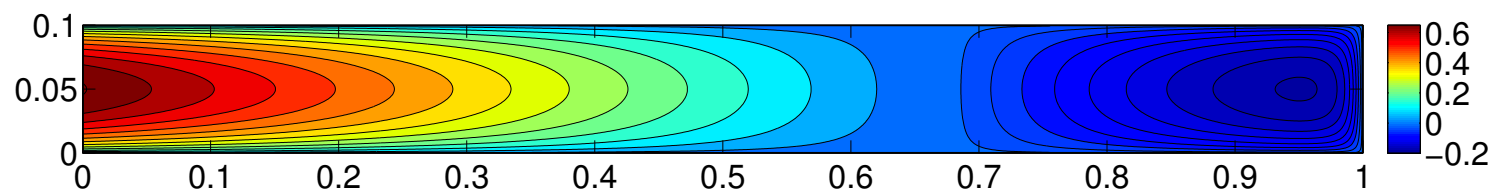

(d)

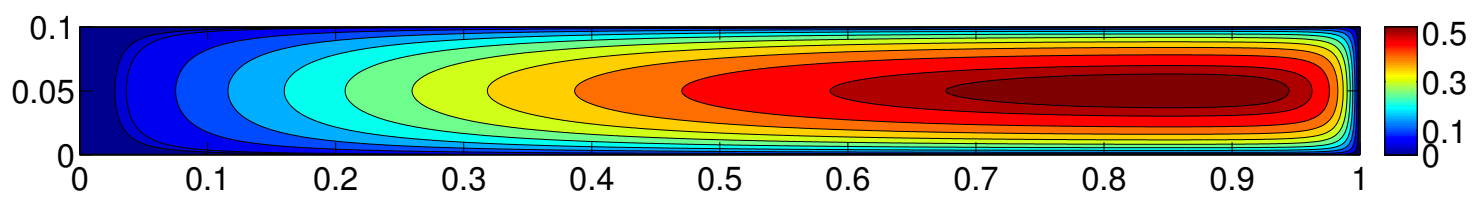

Figure 4: Real and imaginary parts of numerical (a, b) and approximate (c, d) pressure jump distribution. The aspect ratio is $A=0.1$, the reduced frequency is $k=0.5$, and the displacement is the first eigenmode of a clamped-free beam in vacuo. The computation takes about $30 \mathrm{CPU}$ minutes, with $N_{c}=900, N_{s}=90, N_{w}=1800$ (convergence tests have shown that the error is less than $1 \%$ ).

far from the trailing edge. At the trailing edge, $b^{*}$ is exactly zero, which is what is expected from the Kutta condition. In between, in a region whose chordwise length scales as the inverse of the span, the factor $b^{*}$ varies continuously between 1 and 0 . In other words, the present approximate solution, given by Eq. (31), is equivalent to Lighthill's slender-body theory far from the trailing edge, but a correction is introduced near the trailing edge to fulfill the Kutta condition. We will now try to assess the benefits of this approach by comparing its results to the asymptotic solution introduced in section 2 and to the numerical calculations obtained through a panel method described in section 3 .

\section{Comparison between numerical and analytical results}

\subsection{Generic fluid-structure interaction problem}

To assess the validity of the approximate solution, given in Eqs. (30) and (31), we consider a flexible plate with prescribed lateral motion. The leading edge of the surface is clamped at $X=0$, while the trailing edge is free at $X=1$ (Fig. 1). We choose the lateral displacement to be the first eigenmode of a clamped-free Euler-Bernoulli beam in vacuo (Païdoussis, 1998, 2004). The reduced frequency is defined as $k=\Omega L / U$ and, for simplicity, the pressure jump will be normalized by $\rho U^{2}$. The aspect ratio of the flexible plate is first set to $A=0.1$. The distribution of real and imaginary parts of pressure jump derived respectively from the panel method (section 3) and the approximate solution (Eq. 30) are plotted in Fig. 4.

Focusing first only on the real parts of the pressure jumps (panels a and c in Fig. 4), we can see that the numerical and approximate pressure jump both exhibit an elliptical distribution in the spanwise direction, with zero pressure jump at the side edges $(Y=0$ or $Y=0.1)$. A leading edge singularity is clearly visible in the numerical results (Fig. 4a), but not apparent in the approximate solution (Fig. 4c). Apart from this discrepancy near the leading edge $(0 \leq X \leq 0.1)$, both approaches give similar results. The same conclusion can be reached for the imaginary parts of pressure jumps (panels $b$ and d in Fig. 4). 

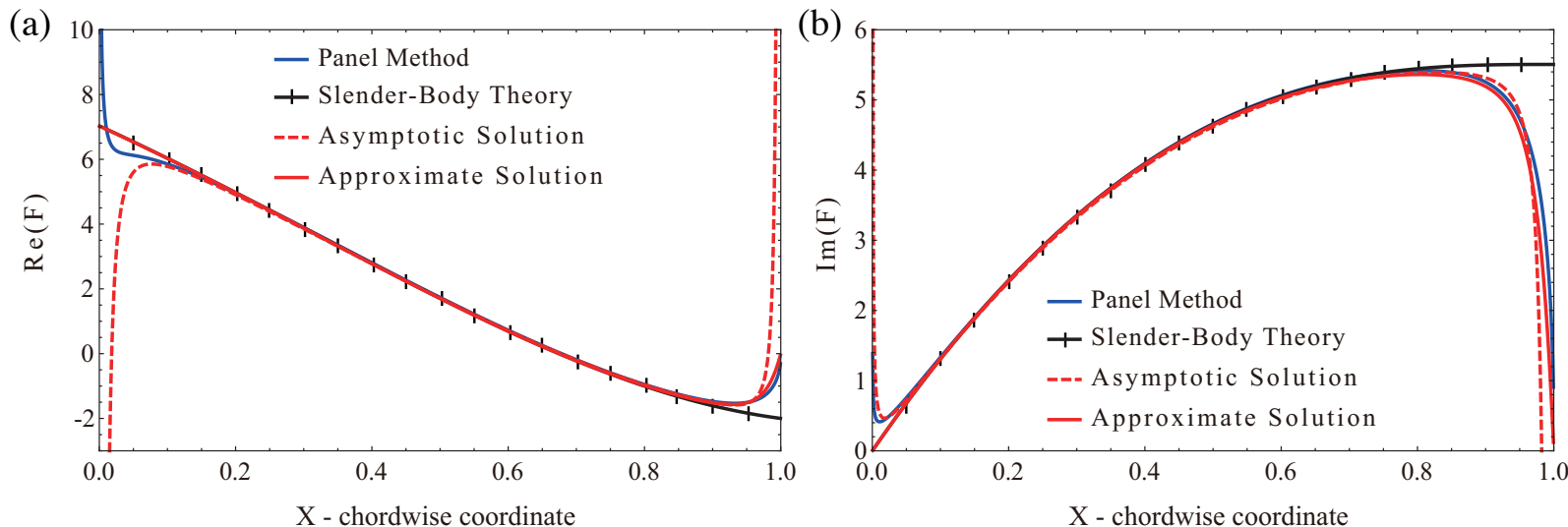

Figure 5: Real (a) and imaginary (b) parts of the force per unit length. The parameters are the same as in Fig. 4.

To reach a more quantitative comparison, we now compare the results of the panel method (section 3), slender-body theory (Lighthill, 1960), the asymptotic solution (section 2), and the approximate solution (section 4), by plotting the chordwise force distribution (Fig. 5), now normalized by $\rho \pi H^{2} U^{2} /(2 L)$ since it is a force per unit length. For this relatively small aspect ratio, all four approaches give similar results in the interval $0.1 \leq X \leq 0.9$. Near the trailing edge, we see that the approximate solution gives the best result, with a force that is very close to the numerical results of the panel method (Fig. 5). In this region, slender-body theory is off, in particular because it does not fulfill the Kutta condition (i.e. $F(X=1)=0$ ), and the asymptotic solution gives a good result except very close to the trailing edge, where it diverges.

Near the leading edge, the asymptotic solution shows the best agreement with the numerical solution, while slender-body theory and the approximate solution give a similar solution, which was expected since $b^{*} \approx 1$ there (see discussion in section 4 ). The singularity at the leading edge results from a suction effect that could be probably be added in the model (Lighthill, 1970; Wu, 1971; Paraz et al., 2016). Note that this inverse-square-root singularity is integrable and does not play a major role in most applications since the leading edge is clamped and the aerodynamic loads applied near the trailing edge thus do negligible work. Note also that this singularity does not pose any problem for the numerical panel method thanks to its integrability.

To evaluate the pros and cons of the approximate solution for moderate aspect ratios, we now consider the same lifting-surface problem but with a larger aspect ratio: $A=0.3$. The pressure jump distribution across the plate is plotted in Fig. 6, and the force distribution along the chord is plotted in Fig. 7. In Fig. 6, the comparison between the results of the panel method and the approximate solution leads to similar conclusions as for the smaller aspect ratio (Fig. 4). We find a good agreement except close to the leading edge. This is confirmed by comparing the force distributions in Fig. 7. The main difference with the results of aspect ratio $A=0.1$ (Fig. 5) is that the leading-edge and trailing-edge regions are now wider, which is what is expected since the length of these regions scales as the plate span.

The average thrust generated by the deforming plate can also be used to compare the different methods. Following Lighthill (1960), the thrust is evaluated by first calculating the mean rate of work $\bar{W}$, as the work done by the force $F(X)$ for a displacement of velocity $i \Omega h(X)$ averaged over one period of time

$$
\bar{W}=\frac{\Omega}{2 \pi} \int_{0}^{\frac{2 \pi}{\Omega}} \int_{-L}^{L} i \Omega h(X) F(X) \mathrm{d} X \mathrm{~d} T .
$$

We then calculated the rate of kinetic energy lost into the wake as $\frac{1}{2} \rho \pi H^{2} U W^{2}(L)$. When this power lost in the wake is subtracted from $\bar{W}$, we obtain the useful work of the thrust force $\bar{T} U$, which means that the average thrust force $\bar{T}$ can be obtained through the equation

$$
\bar{T}=\frac{\bar{W}}{U}-\frac{1}{2} \rho \pi H^{2} U W^{2}(L),
$$


(a)

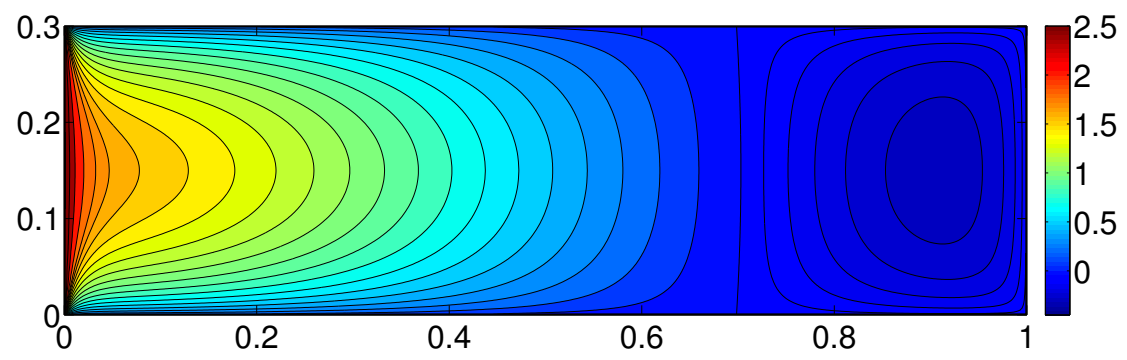

(b)

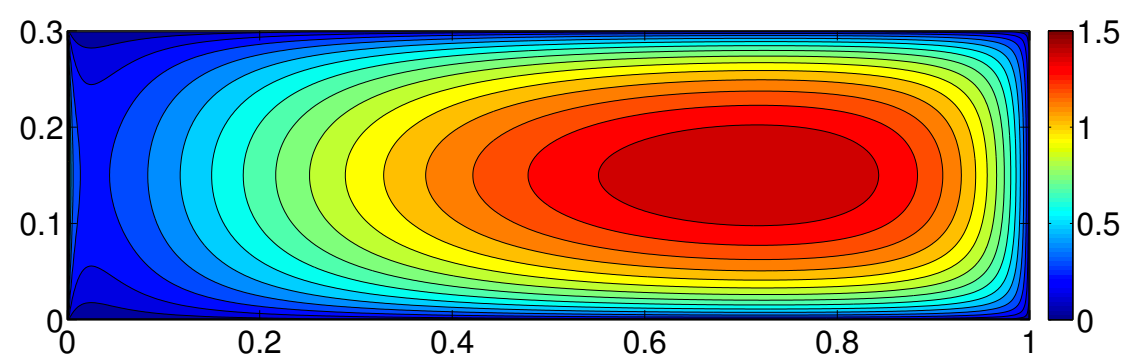

(c)

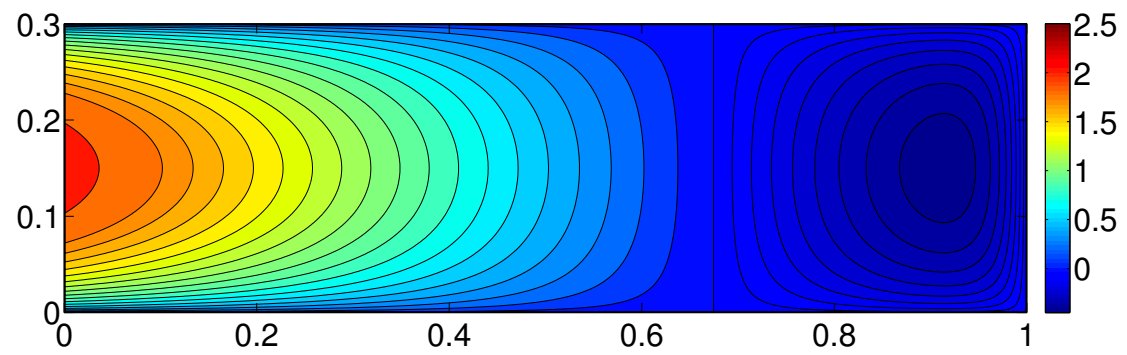

(d)

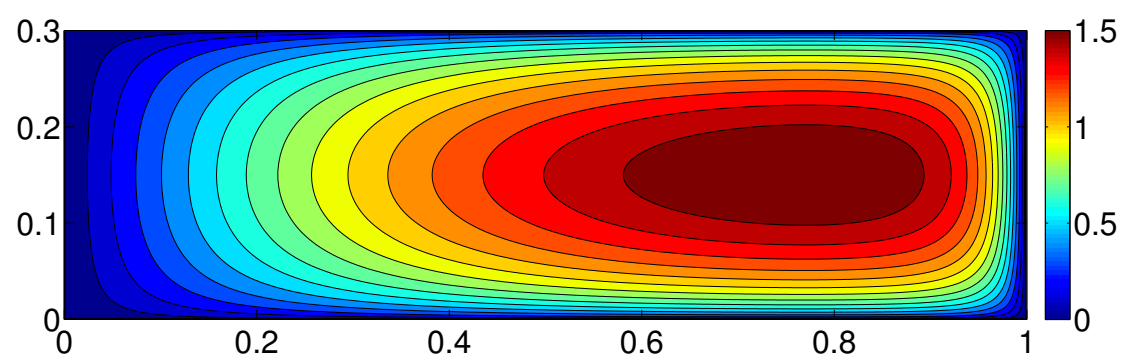

Figure 6: Same as Fig. 4 for an aspect ratio $A=0.3$. The numerical parameters used are: $N_{c}=500, N_{s}=150, N_{w}=1000$.

where $\bar{W}$ is given by Eq. (32). As already noted by Lighthill (1960), in slender-body theory, the thrust has to be calculated through this energy-based approach and cannot be calculated by directly projecting the force along the $x$-direction. We now assume that the lateral displacement of the plate is again the first mode of a clamped-free bream with a reduced frequency $k=10$ (the value $k=0.5$ used in Figs. 4 and 5 could also be used but would yield a negative thrust). Using the formulation of the force given by Eqs. (10) and (31), for respectively the slender-body theory and the proposed approximate method, and inserting it into Eq.(33), the average thrust can be calculated for any aspect ratio. These results are then compared to the numerical results obtained with the paned method in Fig. 8. Note that the asymptotic solution described in $\S 2$ cannot be used to calculate the thrust since the force has non-integrable singularities at the leading and trailing edges. It appears that the approximate solution give better results than slender-body theory. As expected, slender-body theory tends to overestimate the thrust generated because of the finite pressure 
(a)

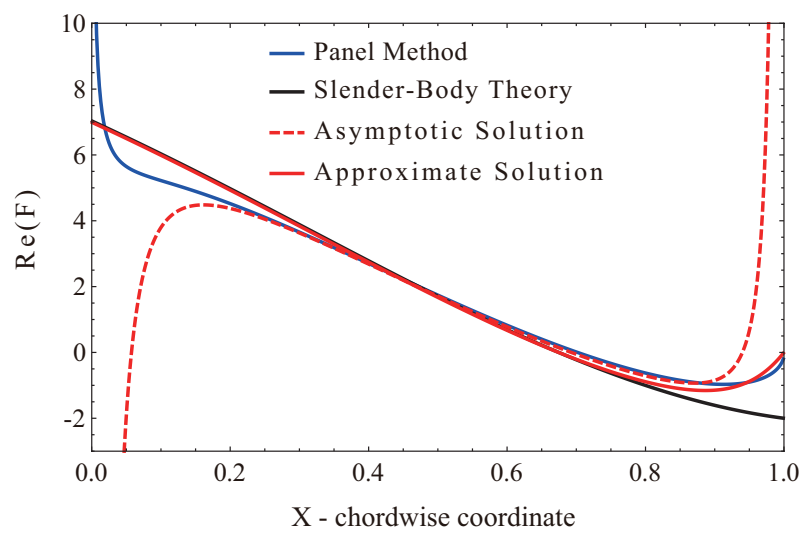

(b)

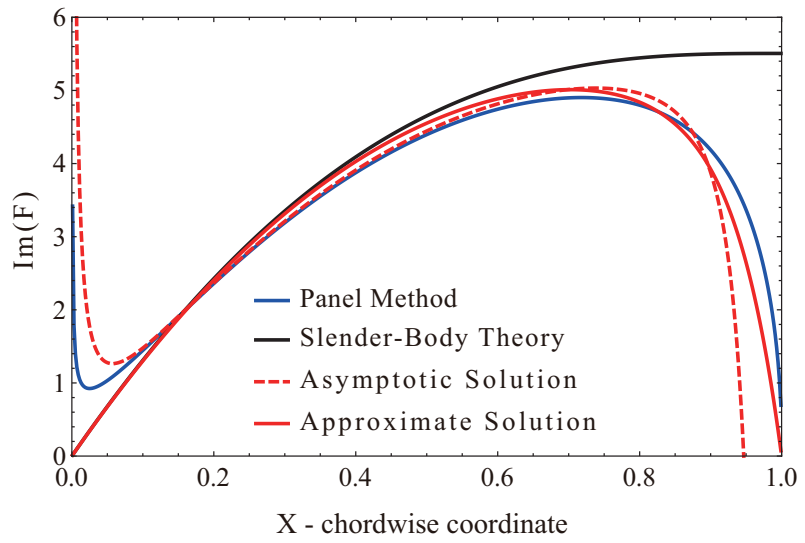

Figure 7: Same as Fig. 5 for an aspect ratio $A=0.3$.

jump at the trailing edge that contributes to the thrust generation. When Kutta condition is enforced, as it is the case in the approximate solution and in the numerical panel method, the pressure jump at the trailing edge is zero and the thrust is notably reduced.

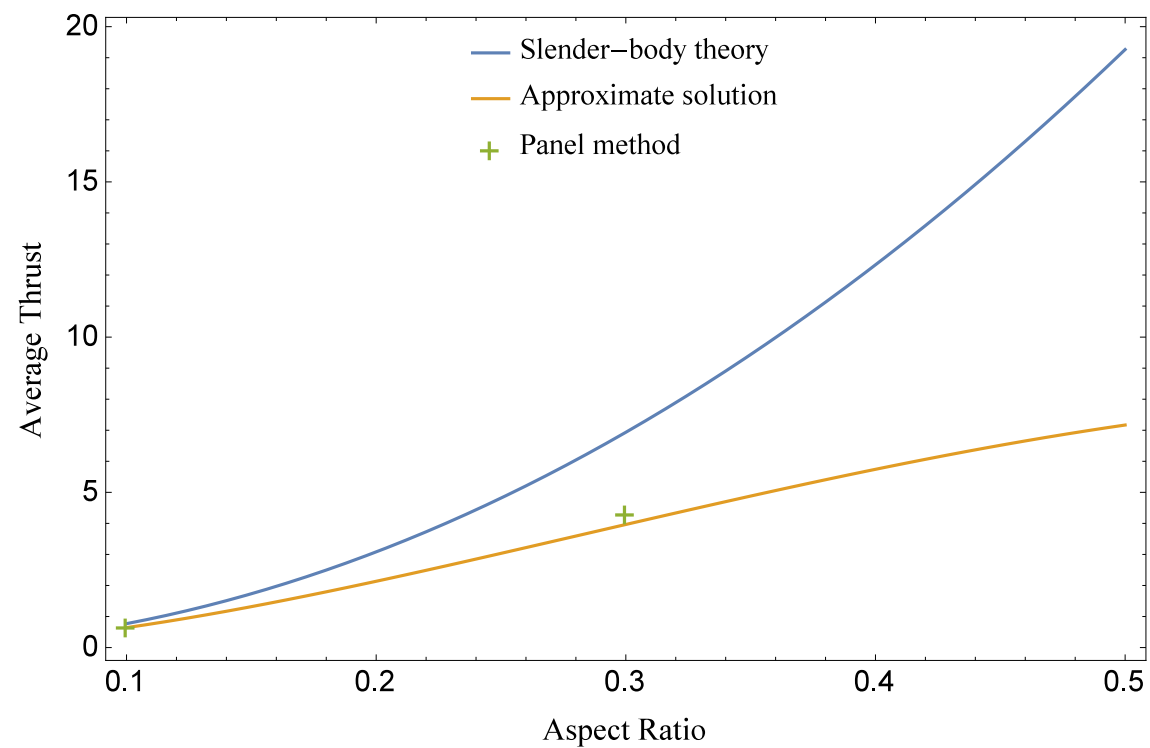

Figure 8: Comparison of the average thrust calculated with the panel method, slender-body theory, and the proposed approximate method. The displacement is the first eigenmode of a clamped-free beam, with reduced frequency $k=10$. Note that the asymptotic method cannot be used to calculate the thrust as it diverges.

These results, and other tests that have been made with different reduced frequencies or different eigenmodes (not reproduced here), give credit to the approximate solution proposed in this paper. This approximate solution describes the aerodynamic loads better than Lighthill's slender-body theory, especially for moderate aspect ratios and near the trailing edge. Although the asymptotic solution may be more rigorous from a mathematical point of view, its numerical implementation is difficult because of the presence of finite-part integrals. In addition, the asymptotic solution diverges at the trailing edge, which may be a problem in some applications. This is why we believe the use of the approximate solution is preferable. Its implementing cost is minimal compared to slender-body theory and the Kutta condition is ensured at the 


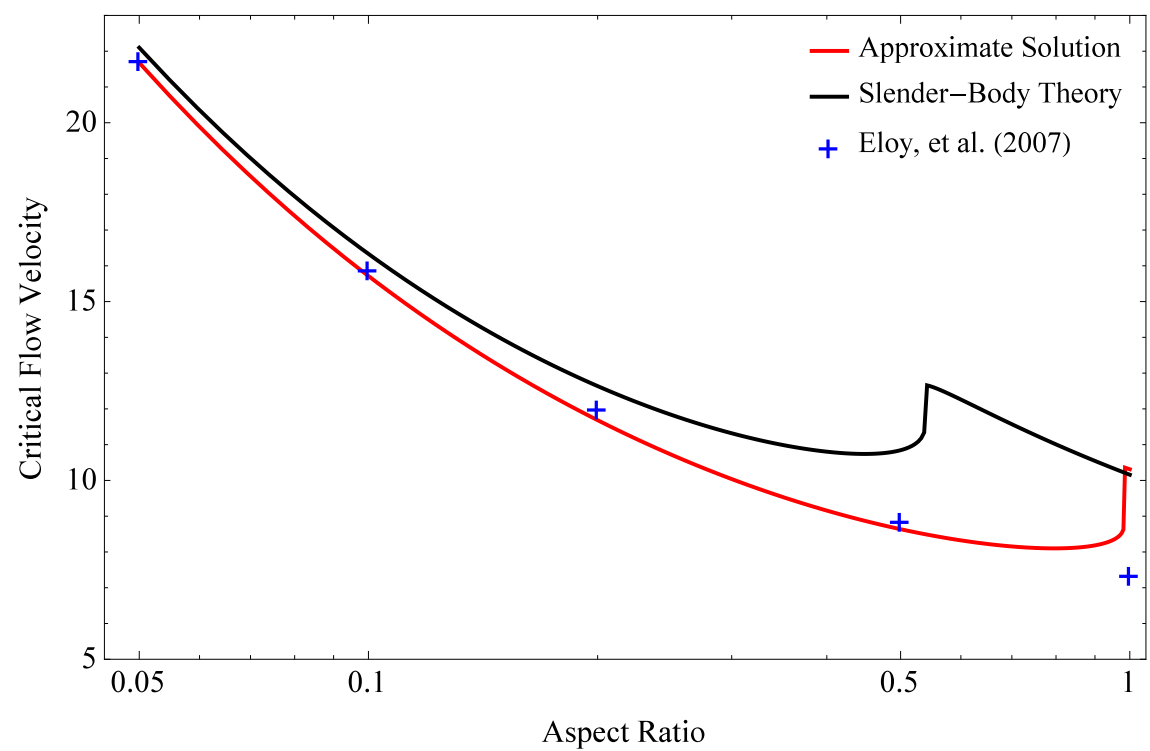

Figure 9: Critical flow velocity $U_{c}^{*}$ as a function of aspect ratio $A$ for a clamped free plate of mass ratio $M^{*}=1$. Crosses are the results from Eloy et al. (2007). The black curve is the marginal stability curve derived with slender-body theory and the red curve shows the result with the approximate solution.

trailing edge.

\subsection{Application to the flag instability}

To further evaluate the interest of the proposed approximate solution for fluid-structure interaction problems, we will now examine its application to the flag instability (Païdoussis, 2004; Shelley and Zhang, 2011). We consider again a clamped-free rectangular plate immersed in a potential flow of incoming velocity $U$ (Fig. 1). Its semi-span is $H$, its semi-chord is $L$, its mass per unit area $\rho_{p}$, and flexural rigidity $D$. We neglect viscoelastic damping and tension due to the skin friction or nonlinear effects.

Following Eloy et al. (2007), we use $2 L, U, 2 L / U, \rho U^{2}$ as the characteristic length, velocity, time, and pressure to make the problem dimensionless. The motion of flexible plate is then governed by the linearized Euler-Bernoulli beam equation

$$
\frac{\partial^{2} z}{\partial t^{2}}+\frac{1}{U^{* 2}} \frac{\partial^{4} z}{\partial x^{4}}+M^{*} F_{0}^{a}=0
$$

where $U^{*}$ is the reduced flow velocity and $M^{*}$ is the mass ratio,

$$
U^{*}=2 \sqrt{\frac{\rho_{p}}{D}} L U, \quad M^{*}=\frac{2 \rho L}{\rho_{p}},
$$

and $F_{0}^{a}$ is the dimensionless force per unit length exerted by the plate on the fluid, which is given by the approximate solution (31).

This linear stability problem can be solved by decomposing the lateral deflection onto the eigenmodes of a clamped-free plate in vacuo. For a mass ratio $M^{*}$, aspect ratio $A$ immersed in a flow of reduced velocity $U^{*}$, the plate is stable if the growth rates of all flutter modes are negative; otherwise it is unstable. The critical velocity $U_{c}^{*}$ is the reduced flow velocity at which at least one flutter mode becomes unstable, when increasing $U^{*}$ from zero.

For a plate of mass ratio $M^{*}=1$, the critical flow velocity is plotted as a function of the aspect ratio $A$ in Fig. 9. The results obtained using slender-body theory and the approximate solution are compared to the results of Eloy et al. (2007) obtained with a numerical spectral method. This comparison shows that 
slender-body theory fails for aspect ratios $A \gtrsim 0.2$, while the approximate solution gives accurate results up to $A \approx 0.7$. This shows that the approximate solution, which can be viewed as a simple correction of Lighthill's slender-body theory, allows to extend the domain of validity up to moderate aspect ratios. Note that the discontinuities of $U_{c}^{*}$ in Fig. 9 (when $A=0.54$ for the black curve, $A=0.98$ for the red one) correspond to shifts of the unstable flutter mode.

\section{Conclusion}

In this paper, we have proposed a method to improve Lighthill's slender-body theory for moderate aspect ratios. We started by recalling how the lifting-surface problem is stated and we gave an asymptotic correction of slender-body theory. We then briefly described a panel method in the frequency domain that has been used to obtain numerical results and compare them with the proposed analytical approaches. Finally, we proposed an approximate solution of the lifting-surface problem that can be viewed as a correction of slender-body theory near the trailing edge.

To assess the benefits of the proposed approximate solution, we applied it to two problems: a generic lifting-surface problem and the flutter instability of a flexible plate (the flag instability). In the generic problem, we compared the pressure jump distribution due to an imposed periodic displacement with the four methods: the panel method, slender-body theory, the asymptotic solution, and the approximate solution. For lifting surfaces of small and moderate aspect ratios, the approximate solution gave superior results, except near the leading edge. In particular, the Kutta condition is fulfilled in the approximate solution, but not in the slender-body theory, nor in the asymptotic solution. In the flag instability problem, we calculated the critical flow velocity with slender-body theory and the approximate solution, and compared those results to those of a spectral method (Eloy et al., 2007). Again, the approximate solution appeared superior to slender-body theory for moderate aspect ratios.

The main contribution of this paper is the approximate solution of the lifting-surface problem given in Eqs. (30) and (31). This approximate solution can be viewed as a correction of Lighthill's slender body theory near the trailing edge that satisfies a Kutta condition. Although the proposed approximate solution is simple to implement and has no extra cost compared to Lighthill's slender-body theory, it appeared to give better results, in particular for moderate aspect ratios. We hope this approach will prove useful and will be applied in fluid structure interaction problems.

\section{Acknowledgments}

Z.Y. acknowledges support from the China Scholarship Council (No. 201204490206).

\section{References}

Albano, E., Rodden, W.P., 1969. A doublet-lattice method for calculating lift distributions on oscillating surfaces in subsonic flows. AIAA J. 7, 279-285.

Alexander, R.M., 1977. Swimming, in: Alexander, R.M., Goldspink, G. (Eds.), Mechanics and energetics of animal locomotion, Chapman \& Hall, London. pp. 222-248.

Anderson, E.J., McGillis, W.R., Grosenbaugh, M.A., 2001. The boundary layer of swimming fish. J. Exp. Biol. 204, 81-102.

Brandao, M.P., 1987. Improper integrals in theoretical aerodynamics - The problem revisited. AIAA J. 25, 1258-1260.

Candelier, F., Boyer, F., Leroyer, A., 2011. Three-dimensional extension of Lighthill's large-amplitude elongated-body theory of fish locomotion. J. Fluid Mech. 674, 196-226.

Candelier, F., Porez, M., Boyer, F., 2013. Note on the swimming of an elongated-body in a non-uniform flow. J. Fluid Mech. $716,616-637$.

Carleman, T., 1922. Sur la résolution de certaines équations intégrales. Almqvist \& Wiksells.

Crighton, D.G., 1985. The Kutta condition in unsteady flow. Ann. Rev. Fluid Mech. 17, 411-445.

Ehrenstein, U., Eloy, C., 2013. Skin friction on a moving wall and its implications for swimming animals. J. Fluid Mech. 718, $321-346$.

Eloy, C., Doaré, O., Duchemin, L., Schouveiler, L., 2010. A unified introduction to fluid mechanics of flying and swimming at high Reynolds number. Exp. Mech. 50, 1361-1366.

Eloy, C., Lagrange, R., Souilliez, C., Schouveiler, L., 2008. Aeroelastic instability of cantilevered flexible plates in uniform flow. J. Fluid Mech. 611, 97-106. 
Eloy, C., Souilliez, C., Schouveiler, L., 2007. Flutter of a rectangular plate. J. Fluids Struct. 23, 904-919.

Guermond, Jean, L., 1987. A systematic formula for the asymptotic expansion of singular integrals. Z. Angew. Math. Phys. $38,717-729$.

Hadamard, J., 1923. Lectures on Cauchy's problem in linear partial differential equations. New Haven Yale University Press.

Hess, F., Videler, J.J., 1984. Fast continuous swimming of saithe (Pollachius virens): a dynamic analysis of bending moments and muscle power. J. Exp. Biol. 109, 229-251.

Katz, J., Plotkin, A., 2001. Low-speed aerodynamics. Cambridge University Press.

Kida, T., Miyai, Y., 1978. An alternative treatment of lifting-line theory as a perturbation problem. Z. Angew. Math. Phys. $29,591-607$

Lauga, E., Powers, T.R., 2009. The hydrodynamics of swimming microorganisms. Rep. Prog. Phys. $72,096601$.

Lemaitre, C., Hémon, P., de Langre, E., 2005. Instability of a long ribbon hanging in axial air flow. J. Fluids Struct. 20, 913-925.

Lighthill, M.J., 1960. Note on the swimming of slender fish. J. Fluid Mech. 9, 305-317.

Lighthill, M.J., 1970. Aquatic animal propulsion of high hydromechanical efficiency. J. Fluid Mech. 44, $265-301$.

Lighthill, M.J., 1971. Large-amplitude elongated-body theory of fish locomotion. Proc. R. Soc. Lond. B 179, $125-138$.

Lighthill, M.J., 1976. Flagellar hydrodynamics: the John von Neumann lecture, 1975. SIAM Rev. , 161-230.

Mangler, K., 1951. Improper Integrals in Theoretical Aerodynamics. Technical Report. British Aeronautical Research Council.

Michelin, S., Doaré, O., 2013. Energy harvesting efficiency of piezoelectric flags in axial flows. J. Fluid Mech. 714, 489-504.

Morse, P.M., Feshbach, H., 1953. Methods of theoretical physics. McGraw-Hill.

Munk, M.M., 1924. The Aerodynamic Forces on Airship Hulls. Technical Report NACA-TR-184. National Advisory Committee for Aeronautics; Washington, DC, United States.

Muskhelishvili, N.I., 1958. Integrals of the Cauchy type, in: Singular integral equations. Springer Nature, pp. 22-55.

Païdoussis, M.P., 1998. Fluid-structure interactions: Slender structures and axial flow, Volume 1. Academic Press.

Païdoussis, M.P., 2004. Fluid-structure interactions: Slender structures and axial flow, Volume 2. Elsevier Academic Press.

Paraz, F., Schouveiler, L., Eloy, C., 2016. Thrust generation by a heaving flexible foil: Resonance, nonlinearities, and optimality. Phys. Fluids 28, 011903.

Shelley, M.J., Zhang, J., 2011. Flapping and bending bodies interacting with fluid flows. Ann. Rev. Fluid Mech. 43, 449-465.

Shirgaonkar, A.A., MacIver, M.A., Patankar, N.A., 2009. A new mathematical formulation and fast algorithm for fully resolved simulation of self-propulsion. J. Comput. Phys. 228, 2366-2390.

Singh, K., Michelin, S., de Langre, E., 2012. Energy harvesting from axial fluid-elastic instabilities of a cylinder. J. Fluids Struct. 30, 159-172.

Tuck, E.O., 1980. Application and solution of Cauchy singular integral equations, in: The application and numerical solution of integral equations. Springer Nature, pp. 21-49.

Videler, J.J., 1981. Swimming movements, body structure and propulsion in cod, Gadus morhua. Symp. Zool. Soc. Lond. 48, $1-27$.

Webb, P.W., 1975. Hydrodynamics and energetics of fish propulsion. J. Fish. Res. Bd Can. 190, 1-158.

Wu, T., 1971. Hydromechanics of swimming propulsion. part 2. some optimum shape problems. J. Fluid Mech. 46, 521-544. 